Rev. Elev. Méd. Vét. Pays trop., 1979, 32 (3) : 263-265.

\title{
Hématologie du taureau N'dama
}

\author{
par L. CHEVRIER $\left(^{*}\right)$
}

\begin{abstract}
RÉSUME
L'auteur rapporte les caractéristiques hématologıques notées lors de l'examen de 10 prélèvements de sang de taureaux N'Dama en provenance de la Côte-d'Ivoire. Il compare les résultats à ceux qui ont été enregistrés au Sénégal, au Togo et au Nigéria et signale le chiffre élevé des numérations leucocytaires en Côte-d'Ivoire.
\end{abstract}

Résultat des examens hématologiques effectués sur 10 taureaux du C. I. A. de Bingerville (Côte-d'Ivoire), appartenant à la SODEPRA, animaux adultes et en bonne santé.

(*) Laboratoire Central de Recherches Vétérinaires, 94703, Maisons-Alfort, Cedex.
Les prélèvements, sur E. D. T. A., ont été effectués par l'ASCEDIA de Maisons-Alfort, transportés par air et examinés le lendemain au Laboratoire Central de Maisons-Alfort.

Les numérations ont été réalisées par comptage électronique (Coulter Counter mod. F. Coultronic S. A.) et les formules leucocytaires établies par la même personne.

\section{RÉSULTATS}

TABLEAU N ${ }^{\circ}$ I - Résultats

\begin{tabular}{|c|c|c|c|c|c|}
\hline \multirow[b]{2}{*}{ Hématies $\left(\times 10^{6} /\right]^{3}$} & \multicolumn{2}{|c|}{ Valeurs extrêmes } & \multicolumn{3}{|c|}{ Moyennes $\pm S d$} \\
\hline & 4,57 & 9,48 & 6,91 & \pm & 1,5 \\
\hline Hémoglobine $\mathrm{g} / 100 \mathrm{ml}$ & 9 & 12 & 10,7 & \pm & 0,9 \\
\hline Hématocrite & 24 & 36 & 31 & \pm & 3,8 \\
\hline $\operatorname{VCM}\left(\right.$ en $\left.\mu^{3}\right)$ & 38 & 58 & 46 & \pm & 6,7 \\
\hline H C M (en $10^{-12}$ ) & 12,7 & 20 & 16 & \pm & 2,4 \\
\hline С С $\mathrm{M}(8 / 100 \mathrm{~m} 1)$ & 31 & 47 & 34 & \pm & 4,3 \\
\hline Leucocytes $\left(\mu^{3}\right)$ & 9760 & 17350 & 13632 & \pm & 2361 \\
\hline G. neutrophiles $\left(\mu^{3}\right)$ & 3978 & 7768 & 6007 & \pm & 970 \\
\hline G. éosinophiles $\left(\mu^{3}\right)$ & 501 & 3804 & 1623 & \pm & 1039 \\
\hline G. basophiles $\left(\mu^{3}\right)$ & 0 & 157 & 31 & \pm & 62 \\
\hline Lymphocytes (\lrcorner$\left.^{3}\right)$ & 3318 & 7981 & 5204 & \pm & 1585 \\
\hline Monocytes $\left(\mu^{3}\right)$ & 0 & 694 & 264 & \pm & 224 \\
\hline G. neutrophiles p. 100 & 30 & 62 & 45 & & \\
\hline G. éosinophiles p. 100 & 4 & 24 & 11,5 & & \\
\hline G. basophiles p. 100 & o & 1 & 0,2 & & \\
\hline Lymphocytes p.100 & 31 & 52 & 41 & & \\
\hline Monocytes p.100 & 0 & 4 & $1, B$ & & \\
\hline
\end{tabular}


Ces résultats sont à rapprocher de ceux qui ont été publiés, dans d'autres pays atricains de l'ouest par CUQ, AKAKPO et FRIOT au Sénégal et au Togo (2) ODUYE et OKUNAIYA au Nigéria (5) FRIOT et CALVET au Sénégal (4). C'est ce qui a été réalisé dans le tableau II qui reproduit les principales valeurs hématologiques relevées par ces auteurs.

TABL. $N^{\circ}$ II-Principales valeurs hématologiques relevées sur les taureaux NDama de l'Afrıque de l'Ouest.

\begin{tabular}{|l|c|c|c|c|}
\hline Nombre d'animaux & $\begin{array}{c}\text { Sênégal } \\
16\end{array}$ & $\begin{array}{c}\text { Togo } \\
12\end{array}$ & $\begin{array}{c}\text { Nigeria } \\
10\end{array}$ & $\begin{array}{c}\text { Côte } \\
\text { Ivoire } \\
10\end{array}$ \\
\hline Hématies T/1 & 8,32 & 7,56 & $/$ & 6,91 \\
$\begin{array}{l}\text { Hémoglobine } \\
\text { g/100 m1 }\end{array}$ & 10,5 & 10,9 & 12,4 & 10,7 \\
Hématocrite & 34 & 34,7 & 38,7 & 31 \\
leucocytes G/1 & 7,14 & 9,15 & 12,4 & 13,6 \\
lymphocytes p.100 & 35 & 47 & 22,8 & 41 \\
G. neutrophiles & 49 & 40 & 60,5 & 45 \\
p.100 & & & & \\
\hline
\end{tabular}

Les valeurs érythrocytaires et celles de l'hématocrite ne diffèrent pas sensiblement entre les animaux N'Dama du Sénégal, du Togo et de la Côte-d'Ivoire. Il est d'ailleurs possible que les faibles différences des moyennes s'inscrivent dans les variations physiologiques normales d'une population et le petit nombre de prélèvements analysés ne permet pas d'amortir les écarts individuels. Les taux d'hémoglobine sont très voisins, confirmant les observations de VOHRADSKY (6) qui estime que ce taux est déterminé par le climat tropical et qu'il est indépendant de la localisation.

Il existe une différence importante dans les numérations leucocytaires. Celles des animaux de la Côte-d'Ivoire sont proches de celles du Nigéria mais s'éloignent des numérations notées au Sénégal et au Togo. Ce point devra être précisé en raison de l'intérêt qu'il présente pour le dépistage des leucoses leucémiques (1).

A l'exception des animaux du Nigéria les pourcentages des lymphocytes se rassemblent entre 35 p. 100 et 47 p. 100 , ce qui peut être considéré comme valable pour un élément cellulaire dont l'établissement implique des variations individuelles normales. Cette stabilité relative permet de fixer les limites des variations individuelles admissibles, notamment pour le diagnostic de la leucose enzootique. Par suite des faibles taux des monocytes et granulocytes éosinophiles, les variations des granulocytes neutrophiles correspondent à celles des lymphocytes.

\section{CONCLUSION}

Les résultats des analyses hématologiques effectuées sur les animaux mâles de la race N'Dama de la Côte-d'Jvoire sont comparables. à ceux qui ont été enregistrés sur les animaux de la même race au Sénégal et au Togo.

Les numérations rouges, les taux d'hémoglobine, l'hématocrite, la répartition des éléments cellulaires ne montrent pas de différences importantes. Par contre, les numérations blanches sont plus élevées et se rapprochent des valeurs notées au Nigéria.

Ce genre d'étude qui trouve son intérêt dans l'établissement des valeurs hématologiques normales d'une catégorie précise de population bovine et, comme conséquence, dans l'appréciation ou le dépistage de certains états pathologiques doit être poursuivi.

\section{SUMMARY}

\section{Hematology of Ndama bull}

The author relates hematological data registered in ten Ndama bulls originating from Ivory Coast. He compares these data to those issued from Senegal, Togo, Nigeria and reports a large number of white blood cells in Ivory Coast animals.

\section{RESUMEN}

\section{Hematologia del toro Ndama}

El autor estudia las características hematologicas resultantes del examen de 10 toros Ndama originarios de la Costa de Marfil y compara sus resultados a los encontrados en Senegal, Togo y Nigeria, señalando la elevada cantidad de leucocitos en los de la Costa de Marfil. 


\section{BIBLIOGRAPHIE}

1. CHEVRIER (L.). Intérêt de l'étude des normes leucocytaires des races bovines en vue du dépistage de la leucose. Bull. Off. int. Epiz., 35e session générale, 1967, $68: 119-131$.

2. CUQ (P.), AKAKPO (A. J.) et FRIOT (D.). Caractéristiques biologiques des hématies des bovins de la zone tropicale de l'Afrique de l'ouest. Rev. Elev. Méd. vét. Pays trop., 1977, 30 (3) : 281-292.

3. CUQ (P.), AKAKPO (A. J.), FRIOT (D.). Les leucocytes des bovins de la zone tropicale de l'Afrique de l'ouest. Rev. Elev. Méd. vét. Pays trop., 1978, 31 (1) : 45-55.
4. FRIOT (D.) et CALVET (H.). Biochimie et élevage au Sénégal. Rev. Elev. Méd. vét. Pays trop., 1973, 26 (4) : $75 a-98 a$.

5. ODUYE (O. O.), OKUNAIYA (O. A.). Haematological studies on the white Fulani and Ndama breeds of cattle. Bull. Epizoot. Dis. Afr., 1971, 19 (3) : 213218.

6. VOHRADSKY (F.). Observation on some blood constituents in british Frisian cattle imported to Ghana. Acta Vet., Brno, 1974, 43 (3) : 221-224. 